\title{
Breathing variability predicts the suggested need for corrective intervention due to the perceived severity of patient-ventilator asynchrony during NIV
}

\author{
Carl Tams $^{1}$ (D) Paul J. Stephan ${ }^{1} \cdot$ Neil R. Euliano $^{1} \cdot$ A. Daniel Martin ${ }^{2} \cdot$ Rohit Patel $^{3} \cdot$ Ali Ataya $^{4} \cdot$ Andrea Gabrielli $^{5}$
}

Received: 12 March 2019 / Accepted: 21 October 2019 / Published online: 29 October 2019

C Springer Nature B.V. 2019

\begin{abstract}
Patient-ventilator asynchrony is associated with intolerance to noninvasive ventilation (NIV) and worsened outcomes. Our goal was to develop a tool to determine a patient needs for intervention by a practitioner due to the presence of patientventilator asynchrony. We postulated that a clinician can determine when a patient needs corrective intervention due to the perceived severity of patient-ventilator asynchrony. We hypothesized a new measure, patient breathing variability, would indicate when corrective intervention is suggested by a bedside practitioner due to the perceived severity of patient-ventilator asynchrony. With IRB approval data was collected on 78 NIV patients. A panel of experts reviewed retrospective data from a development set of 10 NIV patients to categorize them into one of the three categories. The three categories were; "No to mild asynchrony—no intervention needed", "moderate asynchrony—non-emergent corrective intervention required", and "severe asynchrony-immediate intervention required". A stepwise regression with a F-test forward selection criterion was used to develop a positive linear logic model predicting the expert panel's categorizations of the need for corrective intervention. The model was incorporated into a software tool for clinical implementation. The tool was implemented prospectively on 68 NIV patients simultaneous to a bedside practitioner scoring the need for corrective intervention due to the perceived severity of patient-ventilator asynchrony. The categories from the tool and the practitioner were compared with the rate of agreement, sensitivity, specificity, and receiver operator characteristic analyses. The rate of agreement in categorizing the suggested need for clinical intervention due to the perceived presence of patient-ventilator asynchrony between the tool and experienced bedside practitioners was $95 \%$ with a Kappa score of $0.85(\mathrm{p}<0.001)$. Further analysis found a specificity of $84 \%$ and sensitivity of $99 \%$. The tool appears to accurately match the suggested need for corrective intervention by a bedside practitioner. Application of the tool allows for continuous, real time, and non-invasive monitoring of patients receiving NIV, and may enable early corrective interventions to ameliorate potential patient-ventilator asynchrony.
\end{abstract}

Keywords Noninvasive ventilation · Breathing asynchrony $\cdot$ Ventilator monitoring · Work of breathing $\cdot$ Respiratory failure

The study was performed at the UF Health Shands University Hospital in Gainesville Florida. Preliminary results were presented to SCCM conference 2015 in Phoenix Arizona by Carl Tams, PhD.

Electronic supplementary material The online version of this article (https://doi.org/10.1007/s10877-019-00408-7) contains supplementary material, which is available to authorized users.

Neil R. Euliano

neil@conveng.com

Extended author information available on the last page of the article

\section{Introduction}

Non-invasive ventilation (NIV) is an effective therapy treating patients with hypoxia, hypercapnia, and dyspnea associated with respiratory failure [1-9]. A significant benefit of using NIV is to treat and alleviate these symptoms while avoiding complications associated with endotracheal intubation [10]. While NIV has been proven effective for patients with various etiologies [11], it is reported that up to $20 \%$ of patients fail requiring endotracheal intubation $[12,13]$. A common problem that arises during NIV which may contribute to its failure is patient-ventilator asynchrony $[1,12$, 14-19]. Patient-ventilator asynchrony reportedly occurs in $40 \%$ of patients and has been shown to worsen gas exchange, 
waste patient efforts, increase discomfort, and increase the duration of mechanical ventilation [13, 20-22].

Measuring patient-ventilator asynchrony requires detection of patient effort through invasive instrumentation such as an esophageal catheter [23]. Detecting patient-ventilator asynchrony non-invasively can be done by locating the effect of patient effort in the pressure and flow waveforms [24]. However, the effect of patient effort is not always visible in the pressure and flow waveforms. Patient efforts can be hidden from waveforms because of an inadvertent dampening effect caused by leaked flow, which is prevalent during NIV [25]. Even trained clinicians have difficulty detecting patient-ventilator asynchrony when given waveform data in a clinical setting [26, 27]. When patient-ventilator asynchrony is detected, it is unclear at what severity level of asynchrony corrective intervention is beneficial [28].

Based on these findings, detecting patient-ventilator asynchrony non-invasively during NIV is impractical in a routine clinical setting. Thus, there is a need to determine when corrective intervention is beneficial to overcome the effects of patient-ventilator asynchrony.

Our goal was to develop a clinical tool to suggest corrective intervention when assessed by a bedside clinician due to the perceived severity of patient-ventilator asynchrony. We hypothesized a new measure, patient breathing variability, would indicate when corrective intervention is suggested by a bedside practitioner due to the perceived severity of patient-ventilator asynchrony.

\section{Methods}

We first developed a detector to estimate the suggested need for corrective intervention due to the perceived severity of patient-ventilator asynchrony. Then we performed a prospective clinical study evaluating the detector's prediction of the suggested need for corrective intervention against skilled bedside practitioner's assessment.

\subsection{Study patients and data collection}

With approval from the IRB of the University of Florida, adults with respiratory failure from various etiologies receiving non-invasive ventilation (NIV) in the emergency department, surgical intensive care unit, or medical intensive care unit were studied. All patients were ventilated with a dedicated NIV ventilator (Respironics V-60; Philips). Patients were ventilated with the dedicated NIV ventilator per hospital protocol which, in brief, included use of the "S/T" (spontaneous/timed) mode delivering an inspiratory pressure (IPAP) when triggered for inhalation and an expiratory pressure (EPAP) otherwise. Pressure and flow sensors were placed between the breathing circuit tubing and NIV facemask. Data were directed to a respiratory monitor (Respironics NM3, Philips) and recorded on a laptop with proprietary data collection software (Convergent Engineering, Gainesville FL).

\subsection{Phase I-develop the breathing variability detection tool}

A scale with 3 categories was developed to categorize the need for a corrective intervention due to the perceived severity of patient-ventilator asynchrony. The three categories, as seen in Table 1, are (1) no to mild patient-ventilator asynchrony, no corrective intervention required; (2) moderate patient-ventilator asynchrony, non-emergent corrective intervention required; and (3) severe patient-ventilator asynchrony, immediate corrective intervention required to reverse on going or imminent NIV failure.

A panel of eight experts in mechanical ventilation, critical care, and NIV therapy (RRT-5, PhD-2, MD—1) was formed to categorize the need for corrective intervention due

Table 1 Definitions of breathing asynchrony used by clinicians to categorize patient-ventilator asynchrony and the need for corrective intervention are shown

\begin{tabular}{|c|c|c|}
\hline \multicolumn{2}{|r|}{ Patient-ventiltor asynchrony category } & Description \\
\hline 1 & No breathing asynchrony_-patient breathing in phase with ventilator & $\begin{array}{l}\text { Patient appears to be breathing comfortably } \\
\text { Ventilator matches the patient's breathing efforts }\end{array}$ \\
\hline & $\begin{array}{l}\text { Mild breathing asynchrony_-ventilator occasionally out of phase } \\
\text { with patient }\end{array}$ & $\begin{array}{l}\text { Occasional mismatch between patient's breathing pattern and ventila- } \\
\text { tor } \\
\text { Asynchrony does not necessitate changes }\end{array}$ \\
\hline 2 & $\begin{array}{l}\text { Moderate breathing asynchrony_-ventilator frequently out of phase } \\
\text { with patient }\end{array}$ & $\begin{array}{l}\text { Patient appears to be uncomfortable } \\
\text { Apparent mismatch between patient's breathing patterns and ventilator } \\
\text { Asynchrony should be addressed }\end{array}$ \\
\hline 3 & $\begin{array}{l}\text { Severe breathing asynchrony-ventilator always out of phase with } \\
\text { patient }\end{array}$ & $\begin{array}{l}\text { Patient is uncomfortable } \\
\text { Patient is breathing out of phase with ventilator } \\
\text { Difficult to detect breathing } \\
\text { Asynchrony is severe enough to require immediate intervention }\end{array}$ \\
\hline
\end{tabular}


to the perceived severity of patient-ventilator asynchrony. The panel was given 88 waveform data segments, taken retrospectively from 10 patients. The 88 data segments were chosen to represent varying breathing patterns, ranging from consistent to highly variable. The data segments included a 45 s period including: age, weight, gender, diagnosis, ventilator pressure settings, leak flow, ventilator oxygen setting, oxygen saturation, breathing frequency, tidal volume, breathing frequency/tidal volume $\left(\mathrm{f} / \mathrm{V}_{\mathrm{T}}\right), \mathrm{POB} \mathrm{J} / \mathrm{min}$ (power of breathing) [29], minute ventilation, respiratory system compliance [30], total resistance [30] as well as the pressure, flow, and volume waveforms. Each of the experts individually categorized the perceived patient-ventilator asynchrony for the 88 data segments. After which, the experts met and agreed to a single value for each data segment.

A positive linear logistic model was developed using the 88 categorizations of the indication for corrective intervention due to the perceived severity of patient-ventilator asynchrony. The method for developing the model was a stepwise regression with a F-test forward selection criteria [31]. Parameters used in the method were those given to the expert panel with additional parameters that were newly developed for the task. The additional parameters were estimates of breathing patterns and estimates of breathing variability as detailed in online supplemental material 1 . This method used parameters estimating breathing variability to build the model, thus the model was called the breathing variability detection (BVD) algorithm. The BVD algorithm was programmed as a proprietary software package operating on a laptop computer. A laptop with the BVD algorithm and data connection to the ventilator and respiratory monitor comprise the BVD tool.

\subsection{Phase II-breathing variability detection tool implementation}

The BVD tool was attached to a respiratory monitor at a patient's bedside that measured the pressure and flow waveforms at the patient's airway and the NIV ventilator. The tool measured the breathing variability to classify the prospective data into the three categories of suggested need for corrective intervention due to the perceived severity of patientventilator asynchrony.

The BVD was implemented prospectively on 68 patients receiving NIV therapy. Patients were in the medical intensive care unit, surgical intensive care unit, and emergency department. The implementation of the BVD included simultaneous categorization of the need for corrective intervention due to the perceived severity of patient-ventilator asynchrony by the tool and the bedside practitioner. Prior to their evaluation, the bedside practitioners were given a synopsis on patient-ventilator asynchrony as shown in online supplemental material 2 . None of the practitioners evaluating patients were involved in the BVD development phase. The bedside practitioners ( $\mathrm{n}=10$ MDs and 19 RRTs) were blinded to the BVD information, but could use any information available, including: pressure and flow waveform data from a respiratory monitor, cardiovascular data from an attached physiologic monitor, NIV ventilator settings, recent laboratory results, and observed patient agitation or accessory inspiratory muscle use. The practitioners were asked to assess the need for corrective intervention due to the perceived severity of patient-ventilator asynchrony and if the NIV ventilator settings were to be changed, up to a maximum of three evaluations per patient.

The plan for corrective intervention due to the perceived severity of patient-ventilator asynchrony was collected simultaneous to the data measured by the breathing variability detection tool. All data were collected by a study investigator on a laptop, along with patient demographics, ventilator settings, and respiratory monitor parameters.

We calculated the level of agreement of the corrective intervention/asynchrony categories as determined by the bedside practitioner and calculated by the BVD tool with a Cohen's Kappa statistic (K) and a contingency table using a Fischer's exact test. Area under receiving operator characteristic curves was used to assess the predictive ability for categorizing the need for corrective intervention between the bedside practitioner and the algorithm during NIV. Unpaired t-tests were used to compare respiratory measures between the developmental and implementation patient groups, alpha was set at 0.05 for statistical significance.

\section{Results}

In total, data were collected on 78 patients receiving NIV at UF Health between 2012 and 2017. During the first phase of the project, data were collected on 10 patients (development group). During the second phase of the project, data were collected on 68 patients (implementation group). The demographics, ventilator settings and respiratory data were similar between the two populations, as described in Table 2 .

During the development phase, the BVD algorithm was developed. The algorithm uses two parameters to estimate the expert panel's indication for corrective intervention due to the perceived severity patient-ventilator asynchrony. The first parameter estimates the variability of the exhalation time and the second parameter estimates the variability of the breath waveform (online supplemental material 1). All the parameters in the BVD the algorithm are measures of breathing variability, thus the BVD tool calculates asynchrony based on breathing variability, none of the parameters given to the expert panel are used in the BVD tool. While the BVD algorithm was calculated every breath, it was observed that the BVD algorithm did 
Table 2 Patient demographic data, NIV ventilator settings, and patient respiratory data for the study implementation group and the breathing variability detector development group

\begin{tabular}{llll}
\hline & $\begin{array}{l}\text { Implementation } \\
\text { group }(\mathrm{n}=68)\end{array}$ & $\begin{array}{l}\text { Development group } \\
(\mathrm{n}=10)\end{array}$ & $\mathrm{p}$ \\
\hline Demographics & & & 0.50 \\
Age (years) & $61 \pm 15$ & $56 \pm 20$ & 0.28 \\
Weight (lbs) & $198 \pm 86$ & $247 \pm 126$ & - \\
Gender (male/female) & $36 / 32$ & $6 / 4$ & 0.68 \\
Male/female & $53 / 47$ & $60 / 40$ & 0.50 \\
\% Admitting diagnosis with previous respiratory & 56 & 50 & 0.60 \\
$\quad$ disorders (COPD, lung cancer, and cystic fibrosis) & & & \\
$\%$ Other diagnosis* & 44 & 50 & 1.0 \\
NIV ventilator settings & & & 0.13 \\
IPAP (cmH $\left.{ }_{2} \mathrm{O}\right)$ & $13 \pm 5$ & $13 \pm 3$ & 0.03 \\
EPAP (cmH $\left.H_{2} \mathrm{O}\right)$ & $8 \pm 4$ & $6 \pm 2$ & 0.34 \\
FIO $(\%)$ & $0.38 \pm 0.04$ & $0.42 \pm 0.09$ & 0.10 \\
Patient respiratory data & & & 0.26 \\
$\mathrm{SpO}_{2}(\%)$ & $96 \pm 3$ & $95 \pm 3$ & 0.47 \\
f (bpm) & $23 \pm 7$ & $19 \pm 7$ & \\
$\mathrm{~V}_{\mathrm{T}}(\mathrm{L})$ & $0.59 \pm 0.16$ & $0.66 \pm 0.26$ & $12 \pm 4$ \\
$\mathrm{MV}^{(\mathrm{L} / \mathrm{min})}$ & $13 \pm 4$ & & \\
\hline
\end{tabular}

$I P A P$ inspiratory positive airway pressure, EPAP expiratory positive airway pressure, $f$ spontaneous breathing frequency, $M V$ spontaneous minute ventilation

*Other diagnoses in both groups included: Pulmonary embolism, coronary artery disease, pneumonia, pulmonary edema, ketoacidosis, post-extubation respiratory failure, diabetes, myocardial infarction, cholecystectomy, Marfan syndrome, liver abscess, mesenteric ischemia, and perforated bowel not show variation from breath to breath. The parameters in the BVD algorithm are measures of variability over $45 \mathrm{~s}$ which filtered breath to breath variation.

During the implementation phase, 122 bedside evaluations were collected. The need for corrective intervention due to the perceived severity of patient-ventilator asynchrony was correctly categorized by the BVD tool for $95 \%$ of the evaluations (Table 3 ), indicating a highly significant agreement between the tool and the bedside practitioners $[\mathrm{kappa}=0.85(\mathrm{p}<0.001)]$. Sensitivity was $99 \%$ and specificity was $84 \%$ (Table 4 ). The high sensitivity and specificity indicates the BVD tool was able to determine the need for corrective intervention based on the bedside clinician's rating of patient-ventilator asynchrony [Fig. 1, AUC =97\%]. The category "No to mild breathing variability" was the most common observation. However, moderate and severe breathing variability occurred in $19 \%$ of the evaluations (Table 3). Demographic data, ventilator settings, and patients' physiologic response to NIV are listed in Table 1.

It was observed that the BVD was not highly variable during data collection. The BVD is calculated on a breath by breath basis; however, the parameters are both measuring variability of breathing. The variability over a $45 \mathrm{~s}$ period did not change.
Table 3 Contingency table for the category of breathing asynchrony and the need for corrective intervention generated by the breathing variability detector and bedside clinician

\begin{tabular}{cllll}
\hline \multicolumn{5}{c}{ Bedside clinician category } \\
\cline { 2 - 5 } & 1 & 2 & 3 & Total \\
\hline \multicolumn{4}{c}{ Breathing } & variability detector tool category \\
1 & $96(79 \%)$ & $3(2 \%)$ & $0(0 \%)$ & $99(81 \%)$ \\
2 & $2(2 \%)$ & $11(9 \%)$ & $1(1 \%)$ & $14(11 \%)$ \\
3 & $0(0 \%)$ & $0(0 \%)$ & $9(7 \%)$ & $9(7 \%)$ \\
Total & $98(81 \%)$ & $14(11 \%)$ & $10(8 \%)$ & $122(100 \%)$ \\
\hline
\end{tabular}

Category $1-$ no/mild breathing asynchrony with the recommendation for no corrective intervention suggested. Category 2-moderate breathing asynchrony with the recommendation for corrective intervention to improve patient-ventilation interaction. Category $3-$ severe breathing asynchrony with the recommendation for immediate corrective intervention suggested to improve patient-ventilator interaction

Very significant agreement (diagonally italicized cells) between detector generated and clinician generated rankings (Fisher's exact test, $\mathrm{p}<0.001$ ). 116 matched categories (diagonally italicized cells)

Rate of agreement $=\frac{116 \text { matched categories (diagonally shaded cells) }}{122 \text { total obseryations }}=95 \%$. Kappa $=0.85, \mathrm{p}<0.001$ (indicates substantial agreement between the tool and the bedside clinician categories of patient-ventilator asynchrony and the suggested need for corrective intervention) 
Table 4 Performance analysis of the breathing variability detector tool in predicting bedside clinician score of patient-ventilator asynchrony and the need for corrective intervention

\begin{tabular}{|c|c|c|c|c|c|}
\hline & Sensitivity & Specificity & PPV & $\mathrm{ACC}$ & AUC \\
\hline Category $1-$ no/mild breathing asynchrony suggesting no corrective intervention & 98 & 88 & 82 & 96 & 0.997 \\
\hline Category 2 -moderate breathing asynchrony suggesting corrective intervention & 98 & 73 & 91 & 95 & 0.971 \\
\hline Category 3 -severe breathing asynchrony suggesting immediate corrective intervention & 100 & 90 & 93 & 99 & 0.985 \\
\hline Average & 99 & 84 & 89 & 97 & 0.98 \\
\hline
\end{tabular}

$P P V$ positive predictive value, $A C C$ accuracy, $A U C$ area under curve

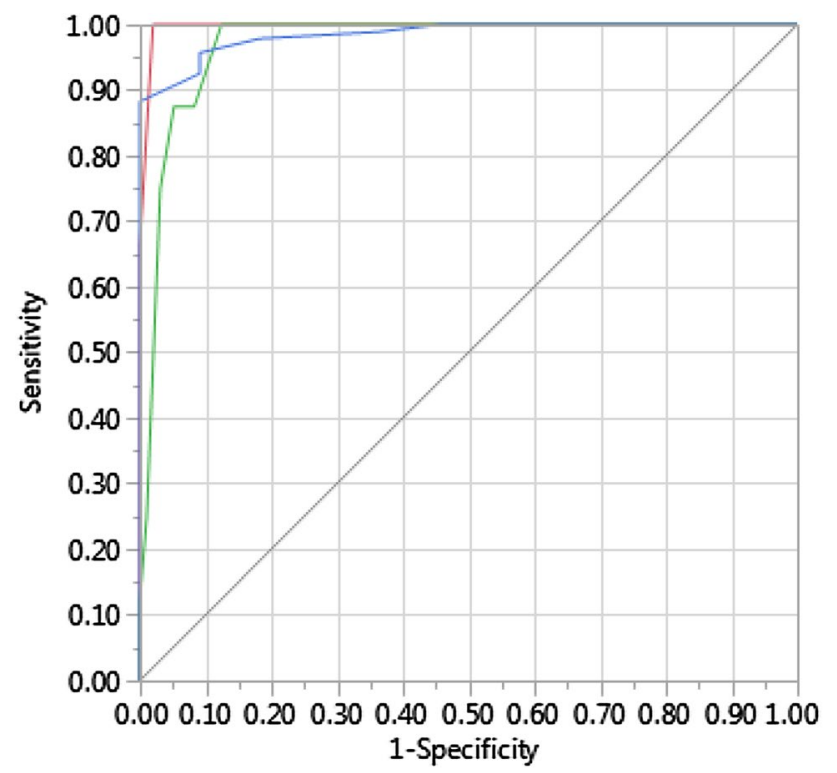

Patient-Ventilator Asynchrony Category

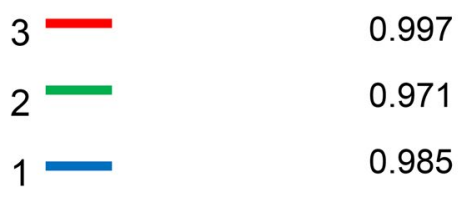

Fig. 1 Areas under the curve for receiver operating characteristic analyses of the breathing variability detector tool for categorizing patient-ventilator asynchrony during non-invasive ventilation are shown. ("1" refers to the tool's ability to correctly classify the asynchrony category of $1-$ no to mild patient-ventilator asynchrony, no corrective intervention required; similarly "2" predicts the asynchrony category of 2-moderate patient-ventilator asynchrony, nonemergent corrective intervention required; and " 3 " predicts the asynchrony category of 3-severe patient-ventilator asynchrony, emergent corrective intervention required to reverse on going or imminent NIV failure)

\section{Discussion}

Our primary goal was to develop a tool to detect when corrective intervention is suggested by a bedside clinician due to the perceived severity of patient-ventilator asynchrony. We hypothesized a new measure, patient breathing variability, would indicate when corrective intervention is suggested. The results indicate that a breathing variability detector can predict a bedside practitioner's suggestion for corrective intervention due to the perceived severity of patient-ventilator asynchrony.

To our knowledge this is the first project designed to detect when practitioner's corrective intervention is required because of patient-ventilator asynchrony. Previous work on this matter has focused on methods measuring patientventilator asynchrony with an Asynchrony Index [24, 32, 33], while others have determined when patients will have successful outcomes [12]. A fundamental flaw in using an Asynchrony Index to determine the need for corrective intervention has been raised; because, the link between a high Asynchrony Index and successful clinical outcomes yet remains uncertain [9]. While the analysis of results from this study are inadequate to ascertain if the breathing variability detector can improve outcomes, it may better describe the patient population that fails NIV. Since the methods in this project are a novel interpretation of patient-ventilatory asynchrony, the discussion on the presented results are limited. Future work will further analyze this novel interpretation of patient-ventilator asynchrony and how it differs from an the Asynchrony Index.

Remarkably, analysis of the results changed our view on how patient-ventilator asynchrony is perceived. During the development phase, we anticipated the detection algorithm would be based on predictors of patient-ventilator asynchrony as found in previous studies; in particular, the quantification of gas leak, and $\mathrm{f} / \mathrm{V}_{\mathrm{T}}$ (rapid shallow breathing index, RSBI) $[15,17,34,35]$. Our original hypothesis during the development phase was that we could develop an algorithm to detect patient-ventilator asynchrony as an expert panel. However, the stepwise regression method determined that the best parameter for detecting patient-ventilator asynchrony was breathing variability (additional results included in online supplement material 1). This result changed our hypothesis for the clinical implementation study to be as stated in the introduction, breathing variability would detect clinical intervention and perceived patient-ventilator asynchrony. We conclude that 
no specific breathing patterns are associated with patient-ventilator asynchrony, which is consistent with previous literature from Longhini et al. [27], but rather the variability in breathing pattern is associated with patient-ventilator asynchrony. Specifically, the events of patient-ventilator asynchrony are the Asynchrony Index (ineffective triggers, double triggers, precycling, and delayed cycling) but the effect of patient-ventilator asynchrony is breathing variability.

The breathing variability detector may be useful as a continuous monitor of NIV patient breathing status and assist the clinician in indicating when to deploy resources to prevent deterioration. Prompt corrective intervention may have a positive clinical impact since it has been reported that early respiratory failure (1-48 h after institution of NIV) is primarily due to progressive deterioration during unsupervised time [19]. If the breathing variability detector can be used to alert a clinician that the effects of patient-ventilator asynchrony are present, the clinician may be able to timely correct the situation [36].

We aknowledge that our study design is limited because the development of the breathing variability tool, the bedside clinician perceptions of patient-ventilator asynchrony, and the need for corrective intervention were all subjective assessments. No invasive measurements of esophageal pressure were recorded; thus, our results cannot be directly compared to the reported Asynchrony Index. Furthermore, Longhini et al. [27] indicated that patient-ventilator asynchrony can't be detected by visual interpretation of the waveforms. Because of this limitation we refer to the patient-ventilator asynchrony measurements as a 'perceived' estimate. We postulated that a clinician can determine when a patient needs corrective intervention due to the perceived severity of patient-ventilator asynchrony. However, while the breathing variability detector appears to be able to determine when the bedside practitioner would act, we have no indication whether the intervention could have resolved the patientventilator asynchrony or improved patient outcomes.

\section{Summary}

In summary, we have showed that experts' bedside evaluation of patient-ventilator asynchrony is likely positive when the breathing pattern is highly variable. A breathing variability detector can interpret non-invasive waveforms and thus continuously suggest when a bedside corrective intervention due to patient-ventilator asynchrony is necessary.

\section{Conclusion}

This project presents a new way to assess patient-ventilator asynchrony. Rather than searching for the occurrence of patient-ventilator asynchrony, as does the Asynchrony
Index, this project evaluates the possibility of searching for the effects of patient-ventilator asynchrony with breathing variability. These positive results merit continued studies to determine if the effects of patient-ventilator asynchrony will correlate, better than other published indexes, with NIV failure.

Acknowledgements We would like to thank Michael Banner for his expert opinion and efforts with the study.

Author contributions All authors offered specific contributions to the production of this manuscript. AG, DM, NE, PS, and CT consulted regularly and collectively to advance the project from start to finish; searched the literature, designed the study, analyzed the data, assisted in preparing the manuscript, and extensively reviewed the manuscript. RP, AA, PS, and CT collected data and added clinical input while reviewing the manuscript. NE and CT analyzed data and performed the statistical analysis.

Funding The project was funded with corporate funding by Convergent Engineering under a contract with Philips HealthCare.

\section{Compliance with ethical standards}

Conflict of interest The authors state no conflict of interest in the research presented in this manuscript. Carl Tams and Neil Euliano are employees of Convergent Engineering and Paul Stephan is a consultant of Convergent Engineering, an engineering firm contracted by Philips HealthCare to perform clinical studies and develop respiratory care technologies. Some authors are listed inventors for a pending patent for detecting patient-ventilator asynchrony in NIV; however, they do not own the patent. The authors have not and do not anticipate receiving additional salaries or compensations for their contribution with this project.

\section{References}

1. Agarwal R, Aggarwal AN, Gupta D, Jindal SK. Role of noninvasive positive-pressure ventilation in postextubation respiratory failure: a meta-analysis. Respir Care. 2007;52(11):1472-9.

2. Burns KE, Adhikari NK, Meade MO. A meta-analysis of noninvasive weaning to facilitate liberation from mechanical ventilation. Can J Anaesth. 2006;53(3):305-15.

3. Keenan SP, Gregor J, Sibbald WJ, Cook D, Gafni A. Noninvasive positive pressure ventilation in the setting of severe, acute exacerbations of chronic obstructive pulmonary disease: more effective and less expensive. Crit Care Med. 2000;28(6):2094-102.

4. Keenan SP, Sinuff T, Cook DJ, Hill NS. Does noninvasive positive pressure ventilation improve outcome in acute hypoxemic respiratory failure? A systematic review. Crit Care Med. 2004;32(12):2516-23.

5. Mehta S, Al-Hashim AH, Keenan SP. Noninvasive ventilation in patients with acute cardiogenic pulmonary edema. Respir Care. 2009;54(2):186-95 discussion 195-197.

6. Nava S, Hill N. Non-invasive ventilation in acute respiratory failure. Lancet. 2009;374(9685):250-9.

7. Ram FS, Picot J, Lightowler J, Wedzicha JA. Non-invasive positive pressure ventilation for treatment of respiratory failure due to exacerbations of chronic obstructive pulmonary disease. Cochrane Database Syst Rev. 2004;1:CD004104. 
8. Weng CL, Zhao YT, Liu QH, Fu CJ, Sun F, Ma YL, Chen YW, He QY. Meta analysis: noninvasive ventilation in acute cardiogenic pulmonary edema. Ann Intern Med. 2010;152(9):590-600.

9. Hess D. Noninvasive ventilation for acute respiratory failure. Respir Care. 2014;58(6):950-72.

10. Burns K, Meade M, Premji A, Adhikari N. Noninvasive ventilation as a weaning strategy for mechanical ventilation in adults with respiratory failure: a Cochrane systematic review. CMAJ. 2014;186(3):E112-22.

11. Nava S. Behind a mask: tricks, pitfalls, and prejudices for noninvasive ventilation. Respir Care. 2013;58(8):1367-76.

12. Nava $S$, Ceriana P. Causes of failure of noninvasive mechanical ventilation. Respir Care. 2004;49(3):295-303.

13. Epstein SK. How often does patient-ventilator asynchrony occur and what are the consequences? Respir Care. 2011;56:25-38.

14. Antonelli M, Conti G, Moro M, Esquinas A, Gonzalez-Diaz G, Confalonieri M, Pelaia P, Principi T, Gregoretti C, Beltrame F, Pennisi MA, Arcangeli A, Proietti R, Passariello M, Meduri GU. Predictors of failure of noninvasive positive pressure ventilation in patients with acute hypoxemic respiratory failure: a multi-center study. Intensive Care Med. 2001;27:1718-28.

15. Berg K, Lang G, Salciccioli J, Bak E, Cocchi M, Gautam S, Donnino $\mathrm{M}$. The rapid shallow breathing index as a predictor of failure of noninvasive ventilation for patients with acute respiratory failure. Respir Care. 2012;57:1548-54.

16. Confaloneiri M, Garuti G, Cattaruzza M, Osborn J, Antonelli M, Conti G, Kodric M, Resta O, Marchese S, Gregoretti C, Rossi A. A chart of failure risk for noninvasive ventilation in patients with COPD exacerbation. Eur Respir J. 2005;25:348-55.

17. Lin M, Guo H, Huang M, Chen C, Wu C. Predictors of successful noninvasive ventilation treatment for patients suffering acute respiratory failure. J Chin Med Assoc. 2008;71:392-8.

18. Moretti M, Cilione C, Tampieri A, Fracchia C, Marchioni A, Nava $\mathrm{S}$. Incidence and causes of non-invasive mechanical ventilation failure after initial success. Thorax. 2000;55:819-25.

19. Ozyilmaz E, Ozsancak A, Nava S. Timing of noninvasive ventilation failure; causes, risk factors, and potential remedies. BMC Pulm Med. 2014;14:19.

20. Blanch L, Villagra A, Sales B, Montanya J, Lucangelo U, Lujan M, Garcia-Esquirol O, Chacon E, Estruga A, Olica JC, Hernandez-Abadia A, Albaiceta GM, Fernandez-Mondejar E, Fernandez R, Lopez-Aguliar J, Villar J, Murias G, Kacmarek RM. Asynchronies during mechanical ventilation are associated with mortality. Intensive Care Med. 2015;41:633-41.

21. Carlucci A, Richard J, Wysocki M, Lepage E, Brochard L. Noninvasive versus conventional mechanical ventilation an epidemiologic survey. Am J Resp Crit Care Med. 2001;163:874-80.

22. De Wit M, Miller K, Green D, Ostman H, Gennings C, Epstein S. Ineffective triggering predicts increased duration of mechanical ventilation. Crit Care Med. 2009;37(10):2740-5.

23. Thille AW, Rodriguez P, Cabello B, Lellouche F, Brochard L. Patient-ventilator asynchrony during assisted mechanical ventilation. Intensive Care Med. 2006;32:1515-22.

24. Chen CW, Lin WC, Hsu CH, Cheng KS, Lo CS. Detecting ineffective triggering in the expiratory phase in mechanically ventilated patients based on airway flow and pressure deflection: feasibility of using a computer algorithm. Crit Care Med. 2008;36:455-61.

25. Ueno $\mathrm{Y}$, Nakanishi N, Oto J, Imanaka H, Nishimura M. A bench study of the effects of leak on ventilator performance during noninvasive ventilation. Respir Care. 2011;56(11):1758-64.

26. Colombo D, Cammarota G, Alemani M, Carenzo L, Barra F, Vachetto R, Slutsky AS, Della Corte F, Navalesi P. Efficacy of ventilator waveforms observation in detecting patient-ventilator asynchrony. Crit Care Med. 2011;39(11):2452-7.

27. Longhini F, Colombo D, Pisani L, Idone F, Chun P, Doorduin J, Ling L, Alemani M, Bruni A, Zhaochen J, Tao Y, Lu W, Garofalo E, Carenzo L, Maggiore SM, Qui H, Heunks L, Antonelli M, Nava S, Navalesi P. Efficacy of ventilator waveform observation for detection of patient-ventilator asynchrony during NIV: a multicenter study. ERJ. 2017;3:00075-2017.

28. Vignaux L, Tassaux D, Carteaux G, Roeseler J, Piquilloud L, Brochard L, Jolliet P. Performance of noninvasive ventilation algorithms on ICU ventilators during pressure support: a clinical study. Intensive Care Med. 2010;36:2053-9.

29. Banner MJ, Tams CG, Euliano NR, Stephan PJ, Leavitt TJ, Martin $\mathrm{AD}, \mathrm{Al}-$ Rawas N, Gabrielli A. Real time noninvasive estimation of work of breathing using facemask leak-corrected tidal volume during noninvasive pressure support: validation study. J Clin Monit Comput. 2016;30(3):285-94.

30. Al-Rawas N, Banner MJ, Euliano NR, Tams CG, Brown J, Martin $\mathrm{AD}$, Gabrielli A. Expiratory time constant for determinations of plateau pressure, respiratory system compliance, and total resistance. Crit Care. 2013; 17:R23.

31. Hocking R. The analysis and selection of variables in linear regression. Biometrics. 1976;32(1):1-49.

32. Gutierrez G, Ballarino G, Turkan H, Abril J, De La Cruz L, Edsall C, George B, Gutierrez S, Jha V, Ahari J. Automatic detection of patient-ventilator asynchrony by spectral analysis of airway flow. Crit Care. 2011;15:R167.

33. Mulqueeny Q, Ceriana P, Carlucci A, Fanfulla F, Delmastro M, Nava S. Automatic detection of ineffective triggering and double triggering during mechanical ventilation. Intensive Care Med. 2007;33:2014-8.

34. Sinderby C, Liu S, Colombo D, Camarotta G, Slutsky A, Navalesi $P$, Beck J. An automated and standardized neural index to quantify patient-ventilator interaction. Crit Care. 2013;17:R239.

35. Carlucci A, Pisani L, Ceriana P, Malovini A, Nava S. Patientventilator asynchronies: may the respiratory mechanics play a role? Crit Care. 2013;17:R54.

36. Di Marco F, Centanni S, Bellone A, Messinesi G, Pesci A, Scala R, Perren A, Nava S. Optimization of ventilator setting by flow and pressure waveform analysis during noninvasive ventilation for acute exacerbations of COPD: a multicentric randomized controlled trial. Crit Care. 2011;15:R283.

Publisher's Note Springer Nature remains neutral with regard to jurisdictional claims in published maps and institutional affiliations. 


\section{Affiliations}

\section{Carl Tams $^{1}$ (I) Paul J. Stephan ${ }^{1} \cdot$ Neil R. Euliano $^{1} \cdot$ A. Daniel Martin $^{2} \cdot$ Rohit Patel $^{3} \cdot$ Ali Ataya $^{4} \cdot$ Andrea Gabrielli $^{5}$}

Carl Tams

carl@ conveng.com

A. Daniel Martin

dmartin@phhp.ufl.edu

Rohit Patel

rohitpatel@ufl.edu

Ali Ataya

ali.ataya@medicine.ufl.edu

Andrea Gabrielli

axg1933@med.miami.edu

1 Convergent Engineering, 107 SW 140th Terrace, STE 1, Newberry, FL 32669, USA
2 Department of Physical Therapy, College of Public Health \& Health Professions, University of Florida, Gainesville, FL 32610, USA

3 Department of Anesthesiology and Department of Emergency Medicine, College of Medicine, University of Florida, 1600 SW Archer Road, PO Box 100254, Gainesville, FL 32610, USA

4 Department of Pulmonary, Critical Care and Sleep Medicine, College of Medicine, University of Florida, 1600 SW Archer Road, Gainesville, FL 32610, USA

5 Department of Anesthesiology Perioperative Medicine and Pain Management, University of Miami Health System, 1611 NW 12th Ave (C-301), Miami, FL 33136, USA 